\title{
Classification Method of Motor Imagery EEG Signal Based on Wavelet Packet and Common Spatial Pattern
}

\author{
Manzhen $\mathrm{Ma}^{+}$, Libin Guo, Kuifeng $\mathrm{Su}$ \\ Department of Control Engineering, Academy of Armored Force Engineering, Beijing 100072, China
}

\begin{abstract}
The traditional EEG signal feature extraction method based on frequency characteristics only extracts the energy feature of each channel, ignoring the correlation information of EEG signals between different channels. To gain a better effect of feature extraction, this article puts forward a feature extraction method based on wavelet packet and common spatial patterns (CSP), namely taking advantage of wavelet packet's multi-resolution characteristics to run the orthogonal decomposition within the all frequency field to extract the motor imagery $\mu$ rhythm and $\beta$ rhythm, from the motor imagery EEG signals of the left hand and the right foot, and further extract the features by doing the spatial filtering through CSP. In combination with the advantages of wavelet packet and CSP, this method could play the relevant information among different channels to the full and classify the two kinds of motor imagery EEG signals by making use of support vector machine (SVM). Better EEG classification results achieved via this experiment are that the maximum classification accuracy of $\mu$ rhythm is $92.8571 \%$ and the maximum classification accuracy of $\beta$ rhythm is $93.5714 \%$. To achieve the practical application of BCI standards.
\end{abstract}

Keywords: brain computer interface (BCI), motor imagery (MI), Wavelet packet, common spatial patterns (CSP)

\section{Introduction}

$\mathrm{BCI}$ is a system that allows the human brain to communicate directly with the external interface[1]. The traditional exchange and control between the human brain and the external environment relies on peripheral nerves and muscle tissue, and BCI provides a direct communication path between the human brain and the external device. Real-time control of the equipment is realized by real-time measuring the brain activity related to the user's intention and converting the activity into the corresponding control signal[2](Fig. 1 for the basic BCI system block diagram). BCI ultimate goal is to form a more natural smooth man-machine communication. This can increase the personnel's special control skills for special equipment, such as divers, astronauts, etc., in certain special environments, as well as reducing personnel workload and improving work efficiency and control accuracy.

The most important part of realizing BCI system based on left-right hand motor imagery is the feature extraction of EEG signal. At present, the main methods of feature extraction are auto regressive (AR) model [3], power spectrum estimation [4], wavelet transform [5] and so on. AR model and power spectrum estimation belong to the frequency domain analysis method, which can not well characterize the time domain information of EEG signal. Wavelet transform is a time-frequency analysis method, although it can analyze the signal in time domain and frequency domain, but it can not have high resolution in both time domain and frequency domain. These methods are to extract the energy feature of each channel itself, ignoring the correlation information of different channels diencephalic electrical signal, EEG feature can not meet the needs of high recognition rate. Therefore, it is very meaningful to explore more effective feature extraction methods.

\footnotetext{
+ Corresponding author. Tel.: + 13263303377.

E-mail address:853856037@qq.com.
} 
In this paper, we propose a method to extract feature EEG signals from left and right footers by means of wavelet packet decomposition and common spatial patterns. When people conduct motor imagery, there will be $8-12 \mathrm{~Hz} \mu$ rhythm and $18-26 \mathrm{~Hz} \beta$ rhythm EEG generated in the brain sensory motor cortex area. When people conduct the unilateral limb motor imagery, the contralateral $\mu$ rhythm and $\beta$ rhythm of the brain will appear amplitude attenuation (ERD); and the ipsilateral $\mu$ rhythm and $\beta$ rhythm of the brain will appear amplitude enhancement(ERS) [6]. According to the ERD / ERS phenomenon, $\mu$ rhythm and $\beta$ rhythm are extracted from the motion imaginary EEG signal by wavelet packet transform to improve the EEG signal-to-noise ratio. And then use the CSP algorithm to extract the characteristics of spatial filtering, fully reflects the correlation between the different channels of information. Finally, the support vector machine (SVM) is used to classify. This study is expected to lay a solid foundation for the further research of online real-time BCI system based on motion imagery.

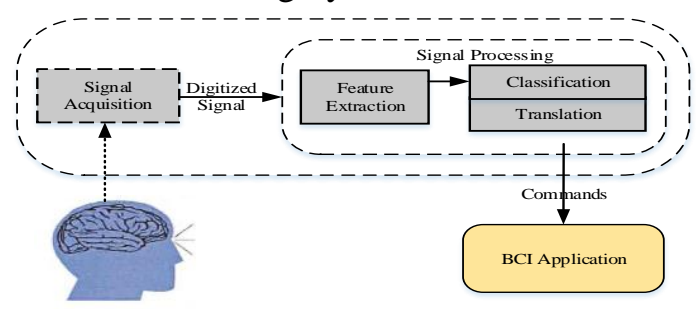

Fig. 1: Basic BCI system block diagram

\section{Fundamentals of Algorithms}

\subsection{The Principle of Wavelet Packet Transform}

Since the EEG signal is very weak ( $\mu \mathrm{V}$ level), and internal mixed with a variety of noise (such as EOG, EMG, ECG, Power Frequency Noise, etc.)[7]. So the signal to noise ratio of EEG is very low. Using wavelet packet transform for EEG signal in frequency domain filtering, can effectively improve the SNR of EEG.

Wavelet packet is developed on the basis of wavelet transform, wavelet packet is more precise than wavelet transform in signal analysis. It can not only carry out the orthogonal decomposition of the signal in the whole frequency band, but also can adaptively select the corresponding frequency band according to the signal characteristics, so that it can be matched with the signal spectrum, and has higher time-frequency resolution [8]. By using the multi-resolution characteristics of wavelet packet transform, we can select the best component combination relation in the original signal of EEG, and then extract the signal range of useful information.

In the wavelet multiresolution signal analysis, the Hilbert space $L^{2}(R)$ can be decomposed into the orthogonal sum of all wavelet subspace $W_{j}(j \in Z)$ according to the different scale factor $i$.

$$
L^{2}(R)=\underset{j \in Z}{\oplus} W_{j}
$$

Wavelet packet on the $W_{j}(j=1,2,3 \cdots)$ in accordance with the binary frequency subdivision, and $U_{j}^{n}$ is the $n$th wavelet subband of the $j$ scale, as shown in Fig.2.

\subsection{Common Spatial Pattern}

The CSP algorithm is applied to the two classification problem, and the optimal spatial filter is constructed by the diagonalization of the covariance matrix of the two kinds of data. After the spatial filtering of the two kinds of data, the difference of the spatial components of the data reaches the maximum, that is to say, the variance of one kind is maximized, and the other one is the minimum variance of [9].

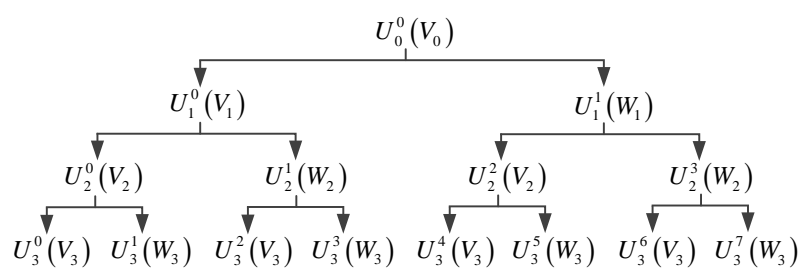

Fig. 2: Wavelet packet decomposition of the 3 layer. 
Single experiment EEG data can be expressed as $N \times T$ matrix $E, N$ is the number of data acquisition channels, $T$ is the number of samples per channel. The normalized spatial covariance of EEG data is shown in equation (2).

$$
C=\frac{E E^{T}}{\operatorname{tr}\left(\mathrm{EE}^{T}\right)}
$$

$\operatorname{tr}(X)$ represents the sum of the diagonal elements of the matrix $X$. The normalized spatial covariance matrices of two kinds of data are denoted by $\mathrm{Cl}$ and $\mathrm{Cr}$ respectively. In order to obtain better spatial filtering effect, the two kinds of experimental data are averaged, and the respective mean normalized spatial covariance matrices $\bar{C} l$ and $\bar{C} r$.

$$
\bar{C} l=\frac{1}{K_{l}} \sum_{i=1}^{K_{l}} C l(i) ; \bar{C} r=\frac{1}{K_{r}} \sum_{i=1}^{K_{r}} C r(i)
$$

$C l(i) 、 C r(i)$ are the normalized covariance matrices of the left and right $i\left(i=1,2, \cdots, K_{l}\right)$-th experimental data, respectively.

The mixed spatial covariance matrix of the two kinds of data is defined as: $C c=\bar{C} l+\bar{C} r$. The $C c$ is decomposed into $C c=U_{c} \lambda_{c} U_{c}, U_{c}$ is the eigenvector of the matrix, $\lambda_{c}$ is the diagonal matrix of the corresponding eigenvalues. The eigenvalues are arranged in descending order, and the whitening transformation is carried out according to the formula (4).

$$
P=\sqrt{\lambda_{c}^{-1}} U_{c}^{T}
$$

After whitening, the characteristic value of $P C c P^{T}$ is equal to 1. For $C l$ and $C r$ to do the following transformation: $S l=P C l P^{T}$ and $S r=P C r P^{T}$. Then $S l$ and $S r$ have a common eigenvector. When $S l=B \lambda_{l} B^{T}$, then $S r=B \lambda_{r} B^{T}$ and $\lambda_{l}+\lambda_{r}=I$, where $\mathrm{i}$ is the unit matrix.

It can be found that the eigenvector corresponding to the largest eigenvalue of $S l$ is the eigenvector corresponding to the minimum eigenvalue of $\mathrm{Sr}$. On the contrary, the eigenvector corresponding to the largest eigenvalue of $S r$ is the eigenvector corresponding to the minimum eigenvalue of $S l$. The projection matrix is obtained by using the eigenvector matrix $B$.

$$
W=\left(B^{T} P\right)^{T}
$$

The experimental data $E$ is decomposed according to the projection matrix $W$, i.e.

$$
Z=W E
$$

Each row vector in $Z$ is a feature vector corresponding to $\lambda_{l}$ or $\lambda_{r}$. Because the eigenvector matrix $B$ is a feature vector corresponding to the descending eigenvalues. The first column in $B$ corresponds to the largest eigenvalue in $\lambda_{r}$, and the last column in $B$ corresponds to the smallest eigenvalue in $\lambda_{r}$. After the transformation of formula (5) and (6), the first line of $Z$ corresponds to the largest eigenvalue in $\lambda_{r}$, and the last line of $Z$ corresponds to the smallest eigenvalue of $\lambda_{r}$. Finally, the variance of each line of $Z$ is used to distinguish the two kinds of data.

\section{Experiment and Result Analysis}

\subsection{Experimental Data}

The experimental data were obtained from the Data sets IV trial B calibration data provided by the third brain machine interface competition in 2005. The experiment uses 118 electrodes, the sampling frequency is $100 \mathrm{~Hz}$, the motor imagery cycle is $3.5 \mathrm{~s}$. The subjects relax and sit comfortably in front of the computer. According to the prompts appear on the screen to exercise imagination, imagine the contents of the left hand movement imagination and right foot movement imagination. A single experiment is divided into the following two links:

- $\quad 0 \sim 3.5 \mathrm{~s}$ subjects performed motor imagery (left / right) according to the arrow direction (left / right) on the computer screen;

- After $3.5 \mathrm{~s}$, the arrow on the screen disappears, the subjects are relaxed and the relaxation time is $1.75 \sim 2.25 \mathrm{~s}$. 
The data set contains 105 times of left hand motion imagination and 105 times of right foot movement imagination, a total of 210 sets of experimental data. The first 35 sets of data from left hand and the first 35 sets of data from the right foot form the training set, and the remaining 140 sets of data form the test set.

\subsection{Using Wavelet Packet to Extract EEG Rhythm}

In the left hand and right foot motor imagery EEG signal analysis, according to the ERD/ERS phenomenon to choose a wavelet packet decomposition, the decomposition of each component in the same level, and has the same sampling rate and the length of the data, which can extract the ${ }^{\mu}$ rhythm and the $\beta$ rhythm of EEG is convenient. Because the sampling frequency of the EEG signal is $100 \mathrm{~Hz}$, according to the Nyquist sampling theorem, the bandwidth of the EEG signal is $50 \mathrm{~Hz}$. The wavelet packet is decomposed into three layers by using the db4 wavelet base to decompose the EEG signal, and the minimum resolution is shown in (7).

$$
\Delta f=50 \mathrm{~Hz} / 8=6.25 \mathrm{~Hz}
$$

Therefore, the wavelet packet decomposition coefficient is reconstructed, and the frequency range corresponding to each subband signal of the obtained wavelet packet is shown in Table 1.

Table 1: Frequency Range Corresponding to Each Subband (unit: Hz)

\begin{tabular}{clcl}
\hline Subband & Frequency Range & Subband & Frequency Range \\
\hline$(3,0)$ & $0 \sim 6.25$ & $(3,1)$ & $6.25 \sim 12.5$ \\
$(3,2)$ & $12.5 \sim 18.75$ & $(3,3)$ & $18.75 \sim 25$ \\
$(3,4)$ & $25 \sim 31.25$ & $(3,5)$ & $31.25 \sim 37.5$ \\
$(3,6)$ & $37.5 \sim 43.75$ & $(3,7)$ & $43.75 \sim 50$ \\
\hline
\end{tabular}

As can be seen from Table 1, $\mu$ rhythm EEG is included in the wavelet Subband (3,1), $\beta$ rhythm EEG is included in the wavelet Subband $(3,3)$. The wavelet packet Subband $(3,1)$ and $(3,3)$ were reconstructed to extract the $\mu$ rhythm and $\beta$ rhythm of EEG. It can remove the interference of the noise and preserve the information of the motor imagery, which provides a powerful guarantee for the feature extraction and classification. Fig. 3 is the original EEG signal for single channel motor imagery and the reconstructed signal of the wavelet packet.

\subsection{Common Space Pattern Extraction Feature}

Using the wavelet packet decomposition method described above, $\mu$ rhythm and $\beta$ rhythm were extracted from 105 groups of left hand motion imagination and 105 group right foot motion imagination EEG data respectively. And then through the CSP algorithm to build a spatial filter, so that motion imagination EEG data after spatial filtering of its spatial components in the energy difference to the maximum. Respectively, to extract the left hand and right foot movement imagination $\mu$ rhythm EEG characteristics and $\beta$ rhythm EEG characteristics. The main steps to extract a feature are as follows:

1) Using the equations (2) and (3), we obtain the average normalized spatial covariance matrices of the first 35 groups of left and right foot motion imagination EEG data respectively;

2) The mixed spatial covariance matrix of two kinds of motion imaginary data is obtained;

3) The projection matrix $W$ is obtained by using equations (4) to (5);

4) The 210 groups of motor imagery EEG data were decomposed by formula (6), and the feature vector matrix $Z$ of each group data was obtained;

5) The variance of each line of the eigenvector matrix $Z$ of each data set is taken as the EEG feature, and each group of motor imagery data contains 118 feature points;

6) Due to the large difference between partial eigenvalues, the eigenvalues are logarithmically transformed to obtain the final characteristics, Fig.4 and Fig.5 is left and right foot motor imagery $\mu$ rhythm and $\beta$ rhythm EEG characteristics. 

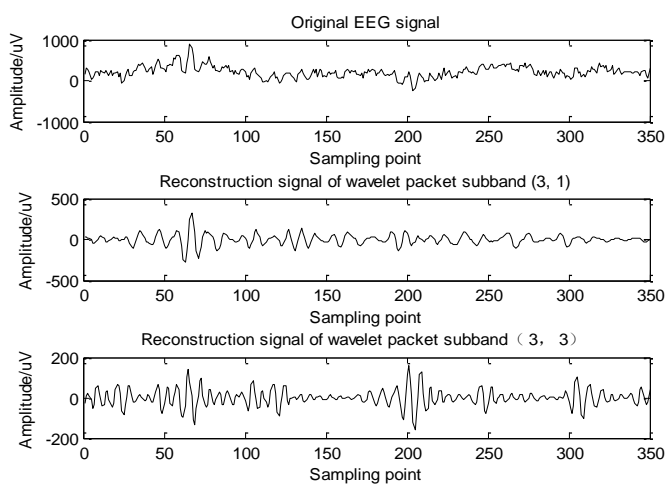

Fig. 3: Single channel motion imagines the original EEG signal, and the wavelet packet subband $(3,1),(3,3)$ reconstructed signal.

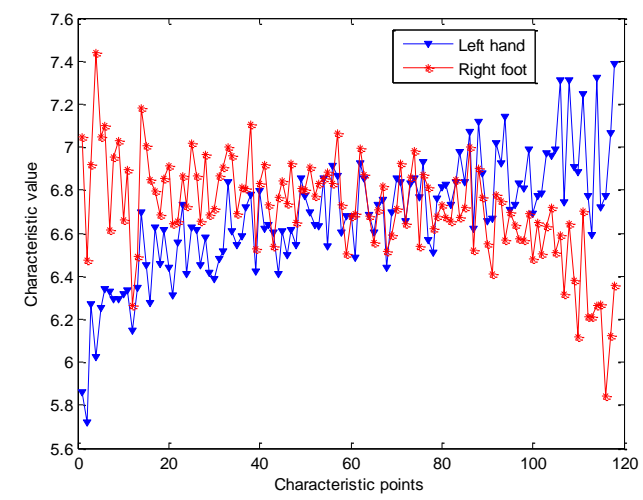

Fig. 4: Left hand and right foot motor imagine $\mu$ rhythm feature distribution.

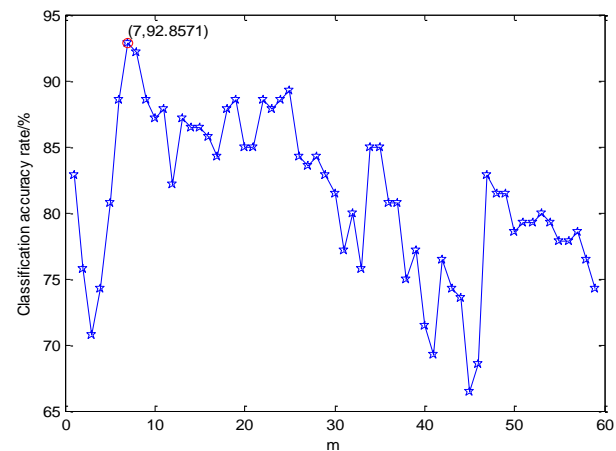

Fig. 6: Curves of $\mu$ rhythm classification accuracy with $m$ value.

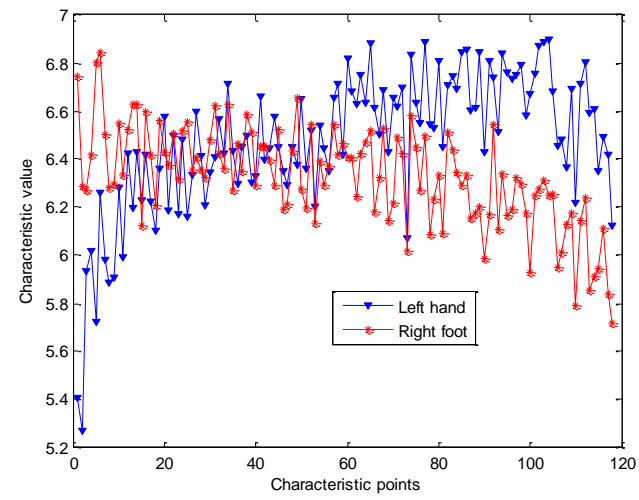

Fig. 5: Left hand and right foot motor imagine $\beta$ rhythm feature distribution.

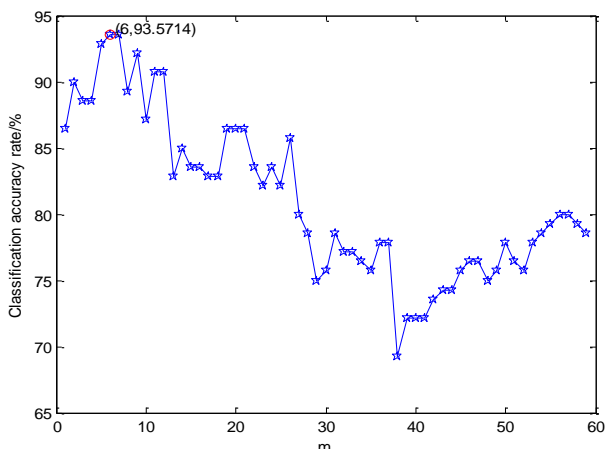

Fig. 7: Curves of $\beta$ rhythm classification accuracy with $m$ value.

\subsection{Support Vector Machine Classification}

SVM was used to classify the two kinds of motor imagery EEG. Because each set of data contains 118 feature points, in order to get the best classification results, we need to select the feature points. As can be seen from Figure 4, the distribution of the eigenvalues of the left hand and right foot is larger at both ends. Therefore, we select the first $\mathrm{m}$ feature points and the rear $\mathrm{m}$ feature points of each set of data to form a feature vector with a length of $2 \mathrm{~m}$ as the input of the classifier. The range of $\mathrm{m}$ is 1 to 59 . The left hand and right foot of the 105 sets of data, respectively, the first 35 groups used for training, the remaining for testing. The formula for calculating the classification accuracy is shown in equation (8).

$$
A_{\text {accuracy rate }}=\frac{L_{\text {correct number }}+R_{\text {correct number }}}{S_{\text {total number }}} \times 100 \%
$$


where $L$ represents the correct number of left hand motor imagery recognition. $R$ represents the correct number of left hand motor imagery recognition. $S$ represents the total number of motor imagery to be identified. The $\mu$ and $\beta$ rhythm EEG classification accuracy with the $\mathrm{m}$ value of the curve as shown in Fig.6 and Fig.7.

As can be seen from Fig.6 and Fig.7: when $m=7$, the classification accuracy of the $\mu$ rhythm EEG reaches a maximum value of $92.8571 \%$. When $\mathrm{m}=6$, the classification accuracy of the $\beta$ rhythm EEG reaches a maximum value of $93.5714 \%$. With the increase of $\mathrm{m}$, the classification accuracy rate decreased gradually, and the change range was $65 \%$ 95\%. The experiment achieved a good classification effect.

\section{Conclusion}

In this paper, the original EEG signal is decomposed by the wavelet packet, and the $\mu$ rhythm and $\beta$ rhythm are extracted. CSP was used to carry out spatial filtering to extract the feature of EEG. SVM was used to classify two kinds of motor imagery EEG with different rhythms. The experimental results show that wavelet packet decomposition and CSP algorithm are combined to extract EEG features. A good combination of the frequency characteristics of the EEG signal and the correlation information between different channels. There were significant differences in EEG features extracted from left hand and right foot. The accuracy rate of $\mu$ rhythm EEG classification was $92.8571 \%$ and the accuracy rate of $\beta$ rhythm EEG classification was $93.5714 \%$. It is proved that the combination of wavelet packet and CSP algorithm can effectively extract the characteristic pattern of motor imagery EEG. It lays the foundation for the further research of online real-time BCI system based on motion imagination.

\section{References}

[1] Daly.L, Muller-Putz.G, et al. Fully Online and Automated Artifact Removal for Brain-Computer Interfacing[C]. IEEE Transactions on Neural Systems and Rehabilitation Engineering. 2015, 23(5): 725-736.

[2] S. Saeedi, R. Chavarriaga, R. Leeb, J. d. R. Millan. Adaptive Assistance for Brain-Computer Interfaces by Online Prediction of Command Reliability[J]. Computational Intelligence Magazine, IEEE. 2016. 11(1): 32-39.

[3] Zou Qing. Feature Extraction and Recognition of EEG Based on AR Model[D]. Central South University. 2008.

[4] Zhang Chong; Zheng Chongxun. Analysis of mental fatigue based on EEG power spectrum[J]. Aerospace Medicine and medical engineering. 2008.21(1).35-39.

[5] Dongying Han; Pei Li; Shujun An; Peiming Shi. Multi-frequency Week Signal Detection Based on Wavelet Transform and Parameter Compensation Band-pass Multi-stable Stochastic Resonance[J]. Mechanical System and Signal Processing. 2016.70-71(000): 995-1010.

[6] Sun Huiyun; Fu Yunfa; Xiong Xin; Yang Jun; Liu Chuanwei; Yu Zhengtao. Research on EEG pattern recognition based on HHT motor imagery[J]. Automation Journal. 2015.41（9）: :1686-1692.

[7] V. Jayaram; M. Alamgir; Y. Altun; B. Scholkopf; M. Grosse-Wentrup. Transfer Learning in Brain-Computer Interfaces[J]. Computational Intelligence Magazine, IEEE. 2016. 11(1): 20-31.

[8] Lei Zhang; Long Zhang; Junfeng Hu; and Guoliang Xiong. Bearing Fault Diagnosis Using a Novel Classifier Ensemble Based on Lifting Wavelet Packet Transforms and Sample Entropy[J]. Shock and Vibration. 2016. 2016(000): 1-14.

[9] Wang Jinjia; Zhang Lingzhi; Hu Bei. Research on feature extraction of multi class kernel space pattern [J]. Journal of Biomedical Engineering. 2012.29(2):217-222. 